\title{
When Robots Engage Humans
}

\author{
John-John Cabibihan • Mary-Anne Williams • \\ Reid Simmons
}

Published online: 25 July 2014

(C) Springer Science+Business Media Dordrecht 2014

One of the earliest recorded robots was a mechanical knight designed by Leonardo da Vinci in the fifteenth century. Driven by mechanical pulleys and cables, this medieval robot could sit, stand, and had basic arm movements. By the end of the twentieth century, the first wave of practical robots was produced to carry out routine assembly and manufacturing tasks on factory floors.

This Special Issue highlights a collection of research articles that shifts the focus from cold, emotionless automata and machines to robots that can create social and emotional connections with their human partners. Here, we present some of the robots that were designed to have engaging human-robot interactions. Their engaging behaviors and features include:

- recognizing the human partner's actions and performing physical motions with associated goals and expectations;

- understanding the human partner's affective states and responding appropriately;

- expressing protest and distress to dissuade the human interaction partner from a potentially unethical course of action;

- displaying human-like facial expressions;

- recognizing and mimicking the body gestures of the human partner;

- providing tactile feedback for richer non-verbal communications;

\section{J.-J. Cabibihan ( $\square)$}

Qatar University, Doha, Qatar

e-mail: john.cabibihan@qu.edu.qa

M.-A. Williams

University of Technology Sydney, Ultimo, NSW, Australia

R. Simmons

Carnegie Mellon University, Pittsburgh, PA, USA
- influencing the perception of the human partner through the robot's gender and the task that the robot is performing;

- encouraging interactive communication on various topics of interest (e.g. poems, music, TV shows); and

- maintaining and respecting social distance.

Due to their humanoid features and affordances, it is expected that robots will be able to engage their human partners. As robots move from the laboratories to our homes, the new normal is robots that are more engaging - the next wave of robotics development. We hope you can benefit from this collection of leading works.

In the first paper, Manipulating Mental States Through Physical Action, Jesse Gray and Cynthia Breazeal investigated how a robot's model of a human's mental state is updated through the robot's visual perception of the surrounding world. The research combines the correct simulations of the immediate future to allow the robot to choose actions that can influence the human partner's mental states. The participants' reaction to the system was evaluated through a competitive game scenario.

In the second article of the edition, Empathic Robots for Long-term Interaction by Iolanda Leite, Ginevra Castellano, André Pereira, Carlos Martinho and Ana Paiva, the authors investigated how robots behave in order to sustain longer interactions with users. They present an empathic model for social robots to interact with children, through the game of chess. The authors measured the children's perception of social presence, engagement and social support when they interacted with the robots in a long term study at an elementary school.

In the third article How Robots Can Affect Human Behavior: Investigating the Effects of Robotic Displays of Protest and Distress, Gordon Briggs and Matthias Scheutz looked 
into the potential of robots to convince a human operator to abandon a potentially unethical course of action in the context of military drones and other ethically sensitive deployment of robots. They conducted an experiment to measure whether a humanoid robot could dissuade a person from performing a task using verbal refusals and affective displays that conveyed distress. Their results show a significant behavioral effect on task completion.

In the fourth paper Implications of Robot Actions for Human Perception. How do we Represent Actions of the Observed Robots?, Agnieszka Wykowska, Ryad Chellali, Md. Mamun Al-Amin and Hermann J. Müller argue that action and perception domains are tightly linked in the human brain. They examined whether robot actions would be represented similarly and, in consequence, elicit similar perceptual effects, as representing human actions. Their results confirm that robot actions elicit perceptual effects of the same kind as human actions.

The fifth article, Deriving Minimal Features for HumanLike Facial Expressions in Robotic Faces, by Casey C. Bennett and Selma Šabanović, reports on a study that explores deriving minimal features for a robotic face to convey information through facial expressions that people can perceive and understand. The study was inspired by recent work in computer vision that has shown that a small number of moving points/lines can be used to capture about $95 \%$ of the information in human facial expressions. They applied those findings to a minimalist robot face design. They ran a series of experiments that explored the effect of various factors, which included added neck motion and degrees of expression. The study addresses fundamental issues about robotic face design and provides hints to develop inexpensive and replicable robotic faces for experimental purposes.

The sixth article, A Framework for User-Defined Body Gestures to Control a Humanoid Robot by Mohammad Obaid, Felix Kistler, Markus Häring, René Bühling and Elisabeth André, proposes a framework that allows users to interact with, and navigate, a humanoid robot using body gestures. The paper presents a taxonomy of the user-defined gesture sets, agreement scores for the gesture sets, and time performances of the gesture motions. Moreover, the study presents a full body interaction system for recognizing the user-defined gestures. The results show that most of the defined gestures can be successfully recognized with a precision between 86-100\% and between 73-96\% accuracy.

The next article, Tactile Interactions with a Humanoid Robot: Novel Play Scenario Implementations with Children with Autism by Ben Robins and Kerstin Dautenhahn, presents a novel robotic skin that provides cognitive mechanisms to improve human-robot interaction capabilities. The article presents two tactile play scenarios developed for robotassisted play for children with autism. The play scenarios were developed against specific educational and therapeutic objectives that were discussed with teachers and therapists. The article presents a detailed description of the play scenarios and case study examples of their implementation in HRI studies between children with autism and the humanoid robot KASPAR.

The eighth article, Keep an Eye on the Task! How Gender Typicality of Tasks Influence Human-Robot Interactions by Dieta Kuchenbrandt, Markus Häring, Jessica Eichberg, Friederike Eyssel and Elisabeth Andre, tested whether the gender typicality of a human-robot interaction task would affect the users' performance of the task. Participants performed either a stereotypically male or a stereotypically female task while being instructed by either a 'male' or a 'female' robot. Results revealed that gender typicality of the task significantly affected the dependent measures: More errors occurred when participants collaborated with the robot in the context of a stereotypically female work domain. Moreover, when participants performed a typically female task with the robot they were less willing to accept help from the robot in a future task and they anthropomorphized the robot to a lower extent. Their findings demonstrate that the gender typicality of HRI tasks substantially influence HRI as well as humans' perceptions and acceptance of a robot.

The ninth article, Acceptability of a Teleoperated Android by Senior Citizens in Danish Society by Ryuji Yamazaki, Shuichi Nishio, Hiroshi Ishiguro, Marco Nrskov, Nobu Ishiguro and Giuseppe Balistreri, explored how Telenoid, a teleoperated android designed as a minimalistic human, can affect people when it is employed to express telepresence and a sense of 'being there'. They reported a pilot test at Danish homes where they found that the elderly with or without dementia showed positive attitudes toward the Telenoid and developed various dialogue strategies. The paper discusses potentials and challenges of the android's embodiment for social inclusion of senior citizens in telecommunications.

The tenth article, Towards a Socially Acceptable Collision Avoidance for a Mobile Robot by Masahiro Shiomi, Francesco Zanlungo, Kotaro Hayashi and Takayuki Kanda, proposes that a mobile robot provide a "social distance" from pedestrians and respect the personal space of the pedestrians. The paper presents a system that implements a human-like collision avoidance model for a mobile robot. The system was tested in 2-h field trials in a real world environment. Results show that the proposed system was perceived as comfortable by pedestrians but also yielded safer navigation than traditional collision-free methods.

Last, but not least, the article Social Robots: Views of Staff of a Disability Service Organization, written by Gregor Wolbring and Sophya Yumakulov, closes this special issue. The paper examines the views of disability service organization workers towards social robotics. The authors conducted a survey of employees of a disability service organization. Many expressed concerns about safety, normality for dis- 
abled people, and artificial interactions. The paper provides key insights for the design of social robots for applications related to persons with disability in order to ensure acceptable and relevant products.

We wish to express our gratitude to Editor-in-Chief Shuzhi Sam Ge and Co-Editor-in-Chief Oussama Khatib, who encouraged us to prepare this special issue. Special thanks to Nathalie Jacobs, Senior Publishing Editor for Engineering at Springer, and to all the reviewers for their support in maintaining the high standard of the journal: A. Sanfeliu, A. K. Pandey, A. Weiss, B. Johnston, C. Meng, C. Laschi, C. Mericli, C. L. Bethel, D. Feil-Seifer, D. Hewson, D. De Tommaso, E. Broadbent, E. Vlachos, G. J. Gelderblom, G. Wolbring, H. Li, H. Osawa, H. Y. Wong, H. He, H. Samani, J. Ham, J. Vitale, J. Vallverdu, J. Fink, K. Poon, L. K. Pinpin, L. Damiano, M. Luber, N. Nasr, R. op den Akker, R. Novianto, S. Sabanovic, T. Park, T. Kanda, W. Wang, W. Guo, Y. K. Tan, Y. Jung, Z. Zhang. 